\section{A modified Verhoeff-van Gieson elastin histochemical stain to enable pulmonary arterial hypertension model characterization}

\author{
K.R. Percival, Z.A. Radi \\ Pfizer Worldwide Research and \\ Development, Drug Safety R\&D, \\ Andover, MA, USA
}

\section{Abstract}

Optimal histochemical staining is critical to ensure excellent quality stained sections to enable light microscopic and histomorphometric image analysis. Verhoeff-van Gieson is the most widely used histochemical stain for the visualization of vascular elastic fibers. However, it is notoriously difficult to differentiate fine elastic fibers of small vasculature to enable histomorphometric image analysis, especially in organs such as the lung. A tissue fixation procedure of $10 \%$ neutral buffered formalin with subsequent fixation in $70 \%$ ethanol further compounds the problem of small vessel staining and identification. Therefore, a modified Verhoeff's elastin stain was developed as a reliable method to optimally highlight the internal and external elastic laminae of small arteries (50-100 $\mu \mathrm{m}$ external diameter) and intra-acinar vessels (10-50 $\mu \mathrm{m}$ external diameter) in $3 \mu \mathrm{m}$ thick lung tissue sections from models of pulmonary arterial hypertension. This modified Verhoeff's elastin stain demonstrated well-defined staining of fine elastic fibers of pulmonary blood vessels enabling subsequent histomorphometric image analysis of vessel wall thickness in small arteries and intra-acinar vessels. In conclusion, modification of the standard Verhoeff-van Gieson histochemical stain is needed to visualize small caliber vessels' elastic fibers especially in tissues fixed in $10 \%$ neutral buffered formalin followed by additional fixation in $70 \%$ ethanol.

\section{Introduction}

Among the various histochemical stains, Verhoeff-van Gieson (VVG) is probably the most widely used histochemical stain for the demonstration of elastic fibers ${ }^{1}$ in pathological conditions such as early lesions of degenerative valvular aortic stenosis, ${ }^{2}$ intimal fibrosis, and aortic atherosclerosis. ${ }^{3}$ The Verhoeff-van Gieson histochemical stain was first described by Ira van Gieson in 1889 as a method of eval- uating collagen fibers in neural tissues. ${ }^{4}$ It was later modified by Frederick H. Verhoeff ${ }^{4}$ as a method to differentiate collagen and other connective tissues and to highlight elastic fibers in particular. ${ }^{5}$ However, while the stain is relatively easy to perform, it is notoriously difficult to differentiate the coarse and fine elastic fibers of blood vessels. ${ }^{6}$ Unlike the elastic fibers of large vasculature that can be easily visualized with VVG, differentiation of fine elastic fibers in small caliber vessels, such as small arteries and intra-acinar vessels of lung affected by pathological conditions such as pulmonary arterial hypertension (PAH), becomes particularly more challenging. In addition to the need for an optimal VVG histochemical stain to visualize such fine elastic fibers in small caliber vasculature, optimal lung tissue fixation and preservation methodology are also critical. Fixation depends on the coefficient of diffusibility of the fixative and the rate at which it reacts with the tissue components. ${ }^{7}$ Ten percent (10\%) neutral buffered formalin (NBF) fixative is used routinely in most histopathology laboratories because of its rapid penetration which is critical to prevent tissue decomposition and to preserve cellular morphology. Other fixatives such as ethanol (EtOH) have low molecular weight and rapid tissue penetration which is thought to contribute to a more uniform tissue fixation and minimize loss of tissue components. ${ }^{7}$ However, rodent lung tissues that were fixed in 10\% NBF for 48 hours followed by 5 days in $70 \% \mathrm{EtOH}$ proved additionally troublesome for demonstrating pulmonary fine elastic fibers of the vessels of interest in PAH rodent models.

The development of PAH is associated with a large spectrum of conditions and diseases, ${ }^{8}$ and its pathogenesis is complicated. ${ }^{9} \mathrm{PAH}$ is characterized by various degrees of remodeling of the pulmonary arterial vessels, which increases the pulmonary vascular resistance and right ventricular afterload, thus contributing to the development of right ventricular dysfunction and failure. ${ }^{8}$ Specific vascular abnormalities associated with PAH include the abnormal appearance of muscle in the walls of normally nonmuscular arteries (i.e., de novo muscularization) (Figure 1) in distal and medial precapillary arteries, loss of precapillary arteries, thickening of large pulmonary arteries, and neointimal formation that is particularly occlusive in vessels less than 100-500 m in external diameter. ${ }^{10}$ Treatments for PAH are limited, and PAH-related mortality remains high. ${ }^{8}$ Elucidating the pathogenesis of PAH continues to be critical for the design of new, effective therapeutic strategies. ${ }^{8}$ Preclinical rodent models of PAH are used to assess the efficacy of emerging therapies. The increase in the extent of the muscularization of the smallest branches of the pulmonary arterial system is the most critical component of PAH
Correspondence: Karen Percival, Pfizer Worldwide Research Development, Drug Safety Research and Development - Necropsy/ Histopathology Laboratories, 1 Burtt Road, Andover, MA 01810, USA.

E-mail: karen.percival@pfizer.com

Key words: Histochemical stain; histomorphology; lung; Verhoeff-van Gieson; elastin.

Contributions: KP, authored the manuscript, codesigned study, performed the tissue processing, microtomy, and elastin staining, developed the modified elastin stain, captured images; ZR, coauthored the manuscript, co-designed study and helped in data interpretation, reviewed the histochemical slides, critically reviewed the manuscript for intellectual content. All authors have read and approved the final manuscript.

Received for publication: 18 November 2015 . Accepted for publication: 25 January 2016.

This work is licensed under a Creative Commons Attribution-NonCommercial 4.0 International License (CC BY-NC 4.0)

(C) Copyright K.R. Percival and Z.A. Radi, 2016 Licensee PAGEPress, Italy

European Journal of Histochemistry 2016; 60:2588 doi:10.4081/ejh.2016.2588

pathogenesis. ${ }^{11}$ Therefore, visualization and quantification of the increased muscularization of pulmonary small and very small arteries in PAH is central to the assessment of the pharmacologic efficacy of new therapeutics. The two most commonly used PAH rodent models are monocrotaline-induced (MCT) PAH and hypoxia-induced PAH. ${ }^{11}$ The 4 key research tools needed to enable optimal visualization and quantification in these PAH models include: i) optimal lung tissue preservation and fixation; ii) sectioning tissues at $3 \mu \mathrm{m}$ thick; iii) a modified Verhoeff-van Gieson histochemical stain to define the fine elastic fibers; iv) histomorphometric image analysis to measure the average wall thickness in both small $(50-100 \mu \mathrm{m})$ arteries and very small (10-50 $\mu \mathrm{m})$ intra-acinar vessels. In this paper, we describe a consistent and reliable modification of the VVG method, which we successfully developed to highlight and visualize the internal and external elastic laminae of small arteries and intra-acinar vessels in lung tissue sections obtained from preclinical efficacy rodent models of PAH.

\section{Materials and Methods}

All animals used in this experiment were housed in a facility accredited by the Association for Assessment and Accreditation 
of Laboratory Animal Care (AAALAC) in accordance with the U.S. guidelines for animal welfare. All animals were euthanized as per standard operating procedures. Lung tissues were collected as per experimental design described below. Lung tissues were obtained from 2 Pfizer-sponsored PAH rodent efficacy models, monocrotaline-induced (MCT) $\mathrm{PAH}$ or Sugen/hypoxia PAH. Methodology of PAH models have been previously described. ${ }^{12,13}$ A third set of lung tissues was obtained from normal Sprague-Dawley rats.

\section{Lung tissue collection, fixation, and processing procedures}

Under isoflurane anesthesia, the rats in the study were exsanguinated, and the pulmonary circulation was flushed with oxygenated $0.9 \%$ saline. The left lung was inflated with $10 \%$ $\mathrm{NBF}$, secured with tied suture at the main stem bronchus, and isolated from the other lobes. The left lobe was immersed in 10\% NBF for 48 $\mathrm{h}$ and transferred to $70 \% \mathrm{EtOH}$ for 5 days before processing. In order to assess whether or not the subsequent fixation in $70 \% \mathrm{EtOH}$ had an effect on staining characteristics, left lungs from the normal rats were collected using the same procedure as described above but were immersed in 10\% NBF for $48 \mathrm{~h}$ only. After fixation, the left lung lobes from both normal and diseased rats were cut transversely into three, 3-4 mm thick sections and routinely processed through graded alcohols and xylene to paraffin blocks. Since we wanted to investigate if tissue section thickness had an impact on the ability to visualize the fine elastic fibers, paraffin blocks were sectioned at $3 \mu \mathrm{m}$, $4 \mu \mathrm{m}$, and $6 \mu \mathrm{m}$. Sections were placed on a flotation bath, picked up on charged slides and air dried for at least $1 \mathrm{~h}$. Air-dried sections were put into a $60^{\circ} \mathrm{C}$ oven for $30 \mathrm{~min}$, deparaf- finized in xylene, and rehydrated through graded alcohols to water. For comparison, the standard VVG (Figure 2) and two modifications to it were conducted on the prepared unstained lung sections. These stains included: i) the standard Verhoeff-Van Gieson elastin stain (Rowley Biochemical, Inc., Danvers, MA, USA) as previously described; 1 ii) the standard Verhoeff stain through differentiation and treatment in sodium thiosulfate (SigmaAldrich, St. Louis, MO, USA) as previously described, ${ }^{1}$ but counterstained for 2 min in $1 \%$ light green (Sigma-Aldrich) solution as report- ed in the literature,${ }^{14}$ in place of the van Gieson counterstain (Rowley Biochemical), to avoid the further differentiating effects of the picric acid in the van Gieson solution; ${ }^{4}$ and iii) a modified Verhoeff's stain with a modified light green counterstain.

\section{Modified Verhoeff's histochemical stain technique}

For the modified Verhoeff's stain with a modified light green counterstain, lung sections were stained in the Verhoeff's staining solution for $30 \mathrm{~min}$ instead of the standard $1 \mathrm{~h}$.

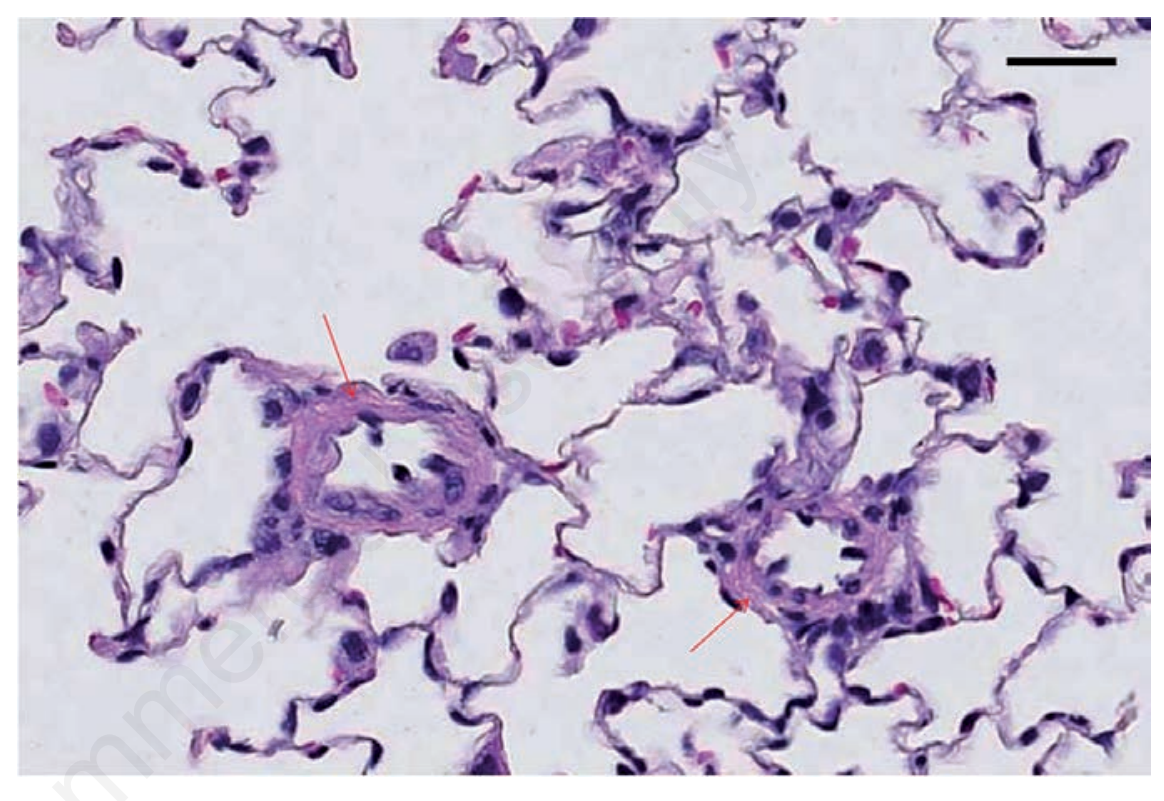

Figure 1. Rat lung tissue section was fixed in $10 \% \mathrm{NBF}$ for $48 \mathrm{~h}$ followed by $70 \% \mathrm{EtOH}$ for 5 days and stained with hematoxylin and eosin, sectioned at $4 \mu \mathrm{m}$. Note small caliber vessels in MCT-induced PAH lung showing the pathology of increased muscularization of small caliber vessel walls (arrows). Scale bars: $25 \mu \mathrm{m}$.

Table 1. Impact of and comparison of fixation procedure and section thickness on 3 histochemical stains.

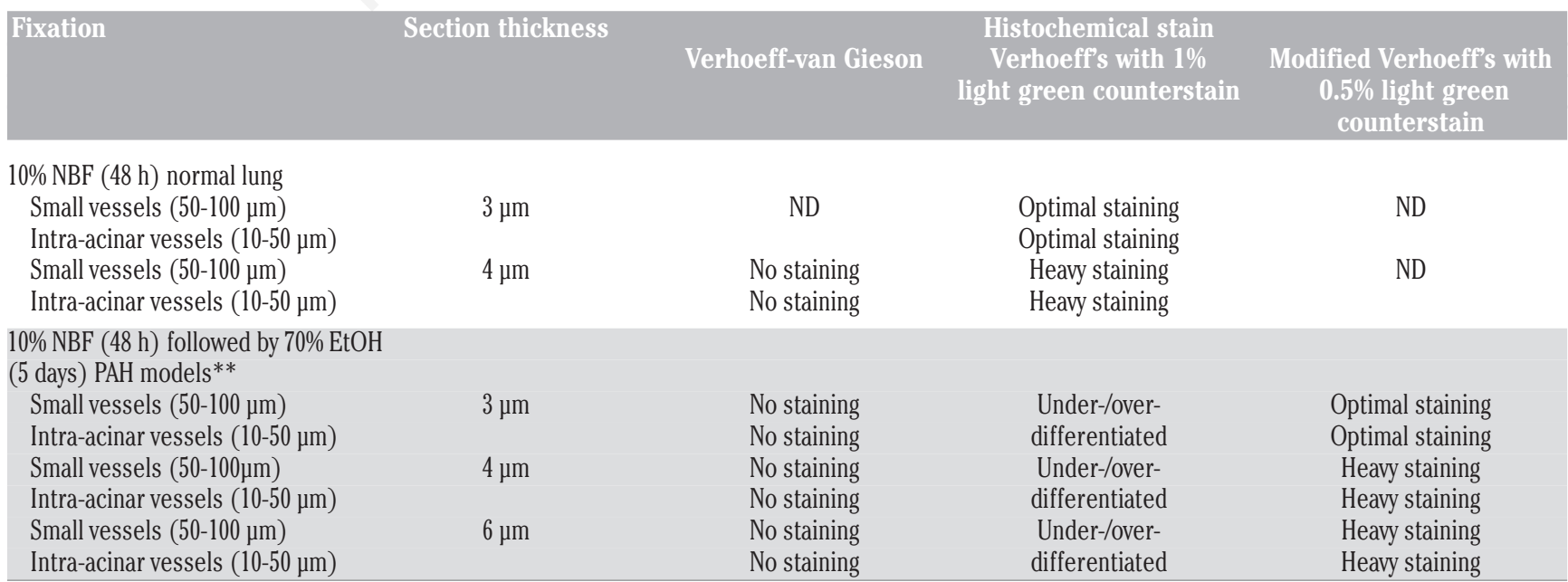

ND, not done;** includes both MCT-induced and Sugen/hypoxia PAH rat models. 
Sections were differentiated in $2 \%$ ferric chloride (Sigma-Aldrich) for $1 \mathrm{~min}$, rinsed briefly in running tap water and checked microscopically for black, sharply defined elastin staining. Slides were returned to $2 \%$ ferric chloride and microscopically checked again at 10-20 s intervals until the background appeared pale violet, and the vessels of interest remained sharply defined. The sections were treated with $5 \%$ sodium thiosulfate for $1 \mathrm{~min}$ and rinsed in running tap water for $5 \mathrm{~min}$. The $1 \%$ light green solution $^{14}$ was diluted to $0.5 \%$. Slides were stained in this solution for $1 \mathrm{~min}$ and rinsed in running tap water for $30 \mathrm{~s}$, dehydrated and cleared through graded alcohols and xylene, and coverslipped.

\section{Results}

A comparison of the findings among the three histochemical stains is presented in Table 1. Under-differentiation (Figure $3 \mathrm{~A}-\mathrm{C}$ ) and over-differentiation (Figure $3 \mathrm{D}-\mathrm{F}$ ) in the Verhoeff technique using a $1 \%$ light green counterstain significantly decreased the ability to clearly visualize the internal and external elastic laminae of small arteries. Under-differentiation produced sections that were too heavily stained for image analysis of vessel wall thickness (Figure 3 A-C). Furthermore, thicker sections (Figure 3 B-C) produced a smudgy appearance making it more difficult to visualize the internal and external elastic laminae. In an effort to clean up the background and remove excess elastin stain, sections were returned to the differentiating solution but quickly became over-differentiated, and the elastin staining of the smaller vessels, as seen in Fgure 3 A-C, was lost (Figure 3 D-F). Regarding section thickness, when comparing $3 \mu \mathrm{m}-, 4 \mu \mathrm{m}$-, and $6 \mu \mathrm{m}$-thick sections stained with the Verhoeff's stain with a 1\% light green counterstain, results from the $3 \mu \mathrm{m}$-thick sections showed the most promise (Figure 3A). The internal and external elastic laminae of some of the smaller vessels from these stained sections, although heavily stained, were somewhat better defined, and some vessel wall thickening was observed. In lung tissue from normal rats sectioned at $3 \mu \mathrm{m}$, no histochemical staining was noted using the standard VVG technique. However, the internal and external elastic laminae of small arteries could be visualized and were well-defined against a light green background using the Verhoeff technique with $1 \%$ light green counterstain (Figure 4). Consequently, efforts were focused on modifying the Verhoeff's staining technique to demonstrate the delicate elastic fibers of the small to very small vessels reliably and consistently in the rodent tissues that had
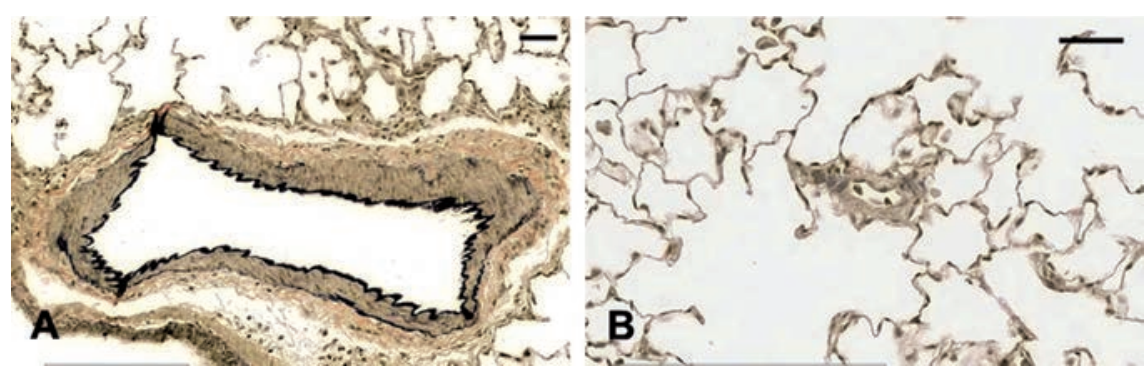

Figure 2. Rat lung tissue sections were fixed for $48 \mathrm{~h}$ in $10 \%$ NBF followed by $70 \%$ EtOH for 5 days and stained with Verhoeff's-van Gieson, sectioned at 3 um, MCTinduced. A) Shows well-defined vessel wall staining of a larger vessel. B) Small caliber vessel shows ill-defined staining of vessel walls. Scale bars: $25 \mu \mathrm{m}$.

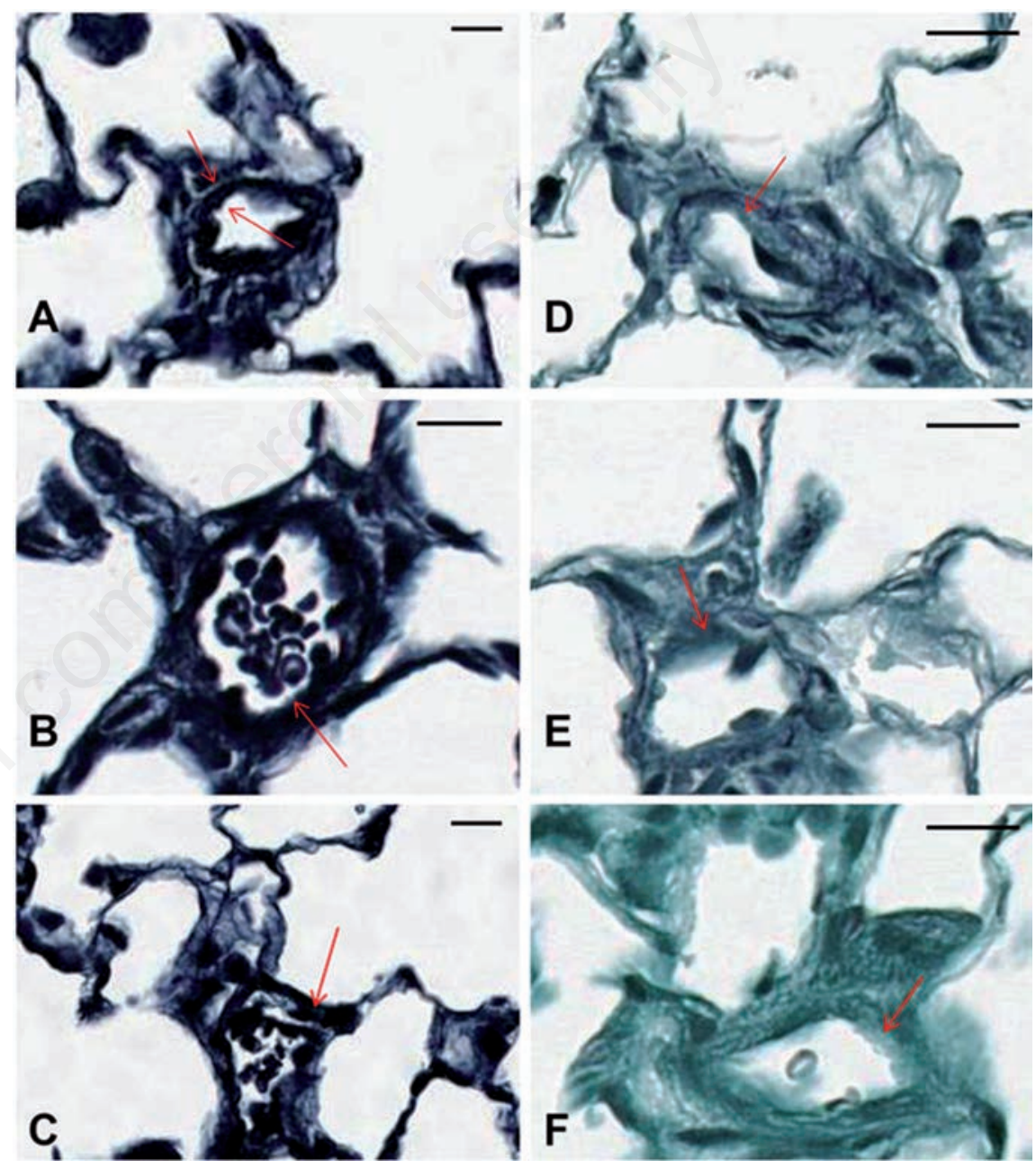

Figure 3. Rat lung tissue sections A-F were fixed in $10 \%$ NBF for $48 \mathrm{~h}$ followed by fixation in $70 \%$ EtOH for 5 days. A) Underdifferentiated Verhoeff stain with $1 \%$ light green counterstain sectioned at $3 \mu \mathrm{m}$ (MCT-induced PAH); arrows show overstained but defined vessels walls with muscle thickening. B) Underdifferentiated Verhoeff stain with $1 \%$ light green counterstain sectioned at $4 \mu \mathrm{m}$ (MCT-induced PAH). C) Underdifferentiated Verhoeff stain with $1 \%$ light green counterstain sectioned at $6 \mu \mathrm{m}$ (MCT-induced PAH); arrows in B and C show overstained and ill-defined vessel walls. D, E, F) Overdifferentiated Verhoeff's stain with $1 \%$ light green counterstain sectioned at 3 $\mu \mathrm{m}, 4 \mu \mathrm{m}$, and $6 \mu \mathrm{m}$, respectively (MCT-induced PAH); arrows show unstained and undefined vessel walls. Scale bars: $25 \mu \mathrm{m}$. 
been fixed with this particular fixation procedure of $48 \mathrm{~h}$ in 10\% NBF followed by 5 days in $70 \% \mathrm{EtOH}$. Therefore, in lung tissue from PAH rats with a fixation procedure of $48 \mathrm{~h}$ in $10 \%$ $\mathrm{NBF}$ followed by 5 days in $70 \% \mathrm{EtOH}$, optimal visualization of the internal and external elastic laminae of small arteries could be obtained if tissue thickness is at $3 \mu \mathrm{m}$ and the modified Verhoeff histochemical staining technique is used (Figure 5).

\section{Discussion}

Generally, any well-fixed tissue will stain satisfactorily with the standard VVG stain, ${ }^{14}$ although $10 \%$ NBF or Zenker solution is the preferred fixative. ${ }^{1}$ In this case, lungs from control, PAH-diseased, or experimental test article-dosed animals were first fixed in $10 \%$ NBF for approximately $48 \mathrm{~h}$ and subsequently transferred to $70 \% \mathrm{EtOH}$ for 5 days. This is a fixation procedure that has been used in $\mathrm{PAH}$ models, and the purpose of this procedure is two-fold: i) minimal fixation time in $10 \% \mathrm{NBF}$ for the preservation of protein epitopes for immunohistochemical (IHC) staining; and ii) standardization of time in fixatives due to multiple study endpoints and variations in the shipping schedule.

The basic principle for the chemical reaction that takes place in the Verhoeff-van Gieson elastin histochemical staining technique involves two main steps. The first step is overstaining, which involves staining the tissue with a regressive hematoxylin consisting of ferric chloride and iodine. ${ }^{4}$ First, the tissue is overstained with a soluble lake of hematoxylin-ferric chloride-iodine. ${ }^{4}$ Both ferric chloride and iodine serve as mordants, but they also have an oxidizing function that assists in converting hematoxylin to hematein. ${ }^{4}$ The second step is differentiation, which is accomplished by using excess ferric chloride mordant to break the tissue-dye-mordant complex. ${ }^{5}$ The dye is attracted to the large volume of mordant in the differentiating solution and is removed from the tissue. ${ }^{5}$ The elastin in the tissue has the strongest affinity to the iron component of the stain ${ }^{5}$ and will retain the dye longer than the other tissue elements, ${ }^{1}$ thereby allowing other elements to be decolorized and the elastic fibers to remain stained. ${ }^{1}$ Sodium thiosulfate removes the excess iodine, and the van Gieson counterstain is used to provide contrast $^{5}$ to the hematoxylin-stained tissue. The resultant product demonstrates elastic fibers and cell nuclei that stain black, collagen that stains red and other tissue components and cell cytoplasm that stain yellow.

Differentiation of any overstaining process is always a necessary, ${ }^{14}$ but precarious step, and Verhoeff's stain is notoriously difficult to differentiate so that the fine elastic fibers can be seen. ${ }^{6}$ Over-differentiation with this stain can lead to a completely colorless background so that a clear yellow counterstain is obtained ${ }^{4}$ leaving tissue elements indistinguishable from each other. Underdifferentiation is actually preferred for this stain, as the picric acid in the van Gieson counterstain will further differentiate the elastic fibers. ${ }^{4}$ Also, the timing of the differentiation step is somewhat dependent on the amount of elastin present in the tissue. ${ }^{4}$ Under-differentiating the Verhoeffvan Gieson stain on these NBF/EtOH-fixed lung tissues produced a gray, muddy background with very ill-defined vessel wall staining. Residual ferric chloride was responsible for the muddy background due to the Verhoeff's stain not being completely removed from the cytoplasm. ${ }^{6}$ In the under-differentiated sections, the picric acid component of the counterstain did not further differentiate the elastic fibers of the smaller vessels well enough for accurate morphometrics. Further differentiation to remove the excess background staining resulted in over-differentiation of the tissue sections and left the elastin fibers of the smaller vessels unstained.

In order to reduce background and excess elastin staining, timing in Verhoeff's solution was decreased from $1 \mathrm{~h}$ to $30 \mathrm{~min}$. Differentiation of sections stained for this shortened time period took about the same amount of time as the $1 \mathrm{~h}$ staining time; however, background staining was significantly cleaner, and the elastic laminae was well stained and defined. Modifying the 1\% light green counterstain to a more dilute solution (0.5\%) produced a lighter background stain
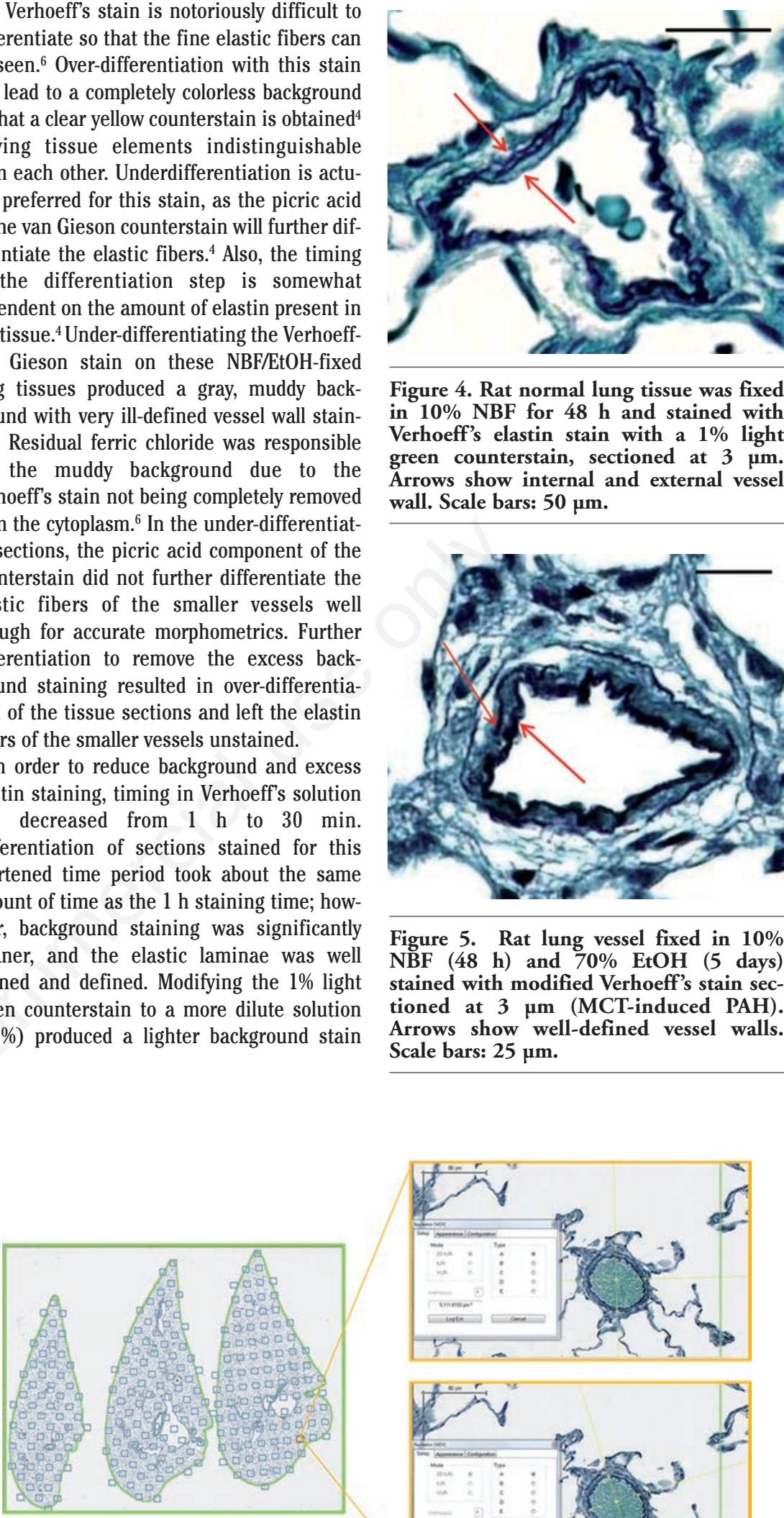

Figure 4. Rat normal lung tissue was fixed in $10 \% \mathrm{NBF}$ for $48 \mathrm{~h}$ and stained with Verhoeff's elastin stain with a $1 \%$ light green counterstain, sectioned at $3 \mu \mathrm{m}$. Arrows show internal and external vessel wall. Scale bars: $50 \mu \mathrm{m}$.

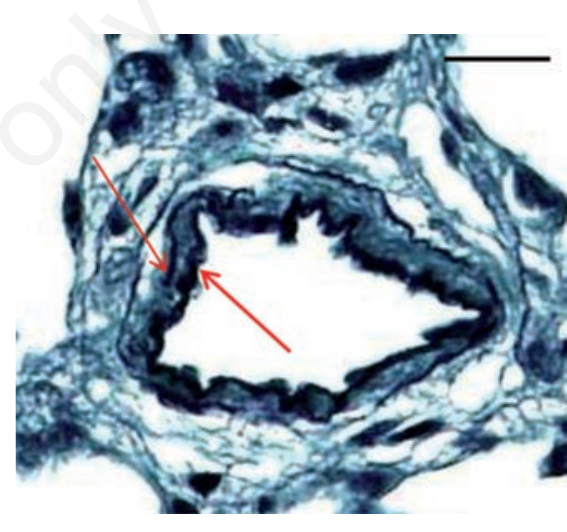

Figure 5. Rat lung vessel fixed in $10 \%$ NBF (48 h) and $70 \%$ EtOH (5 days) stained with modified Verhoeff's stain sectioned at $3 \mu \mathrm{m}$ (MCT-induced PAH). Arrows show well-defined vessel walls. Scale bars: $25 \mu \mathrm{m}$.
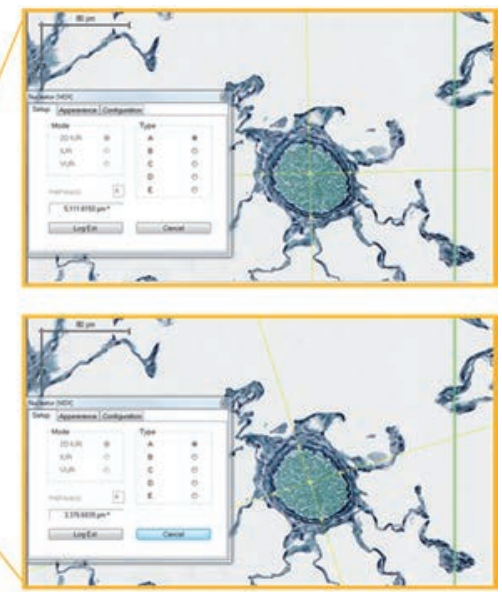

Figure 6. Image analysis methodology of lung sections. Average vessel wall thickness (elastin): random field sampling (Visiopharm newCAST) of $10 \%$ of tissue area with observer-assisted identification of vessels and nucleator probe used to measure wall thickness. 
that provided good contrast to the black elastin stain without overpowering the finer, more delicate fibers. As a result, the well-defined elastin in small caliber vessel walls allowed histomorphometric image analysis of vessel wall thickness to be performed (Figure 6).

\section{References}

1. Carson FL, Hladik C. Histotechnology: A self-instructional text. Chicago, ASCP Press: 2009.

2. Otto CM, Kuusisto J, Reichenbach DD, Gown AM, O'Brien KD. Characterization of the early lesion of 'degenerative' valvular aortic stenosis. Histological and immunohistochemical studies. Circulation 1994; 90:844-53.

3. Taylor HE. The role of mucopolysaccharides in the pathogenesis of intimal fibrosis and atherosclerosis of the human aorta. Am J Pathol 1953;29:871-83.

4. Kazlouskaya V, Malhotra S, Lambe J, Idriss $\mathrm{MH}$, Elston D, Andres C. The utility of elastic Verhoeff-van Gieson staining in dermatopathology. J Cut Path 2013;40: 211-25.

5. Parry N. Re: Verhoeff-van Gieson Stain: A special histology stain for elastic fibers [Web log post - 2014]. Accessed on: March 25, 2015. Available from: http://bitesizebio.com/19952/verhoeff-van-gieson-staina-special-histology-stain-for-elastic-fibers/

6. Ellis RC. Re: Problems in histopathological technique [Web log post - 2002]. Accessed on: June 18, 2014. Available from http://www.ichworld.com/royellis/problems/problem27.htm

7. Srinivasan M, Sedmak D, Jewell S. Effect of fixatives and tissue processing on the content and integrity of nucleic acids. Am J Pathol 2002;161:1961-71.

8. Gomez-Arroyo JG, Farkas L, Alhussaini AA, Farkas D, Kraskaukas D, Voelkel NF, et al. The monocrotaline model of pulmonary hypertension in perspective. Am J Physiol Lung Cell Mol Physiol 2012;302:L363-9.

9. Li XQ, Wang HM, Yang CG, Zhang XH, Han DD, Wang HL. Fluoxetine inhibited extracellular matrix of pulmonary artery and inflammation of lungs in monocrotalinetreated rats. Acta Pharmacol Sin 2011:32:217-22.

10. Rabinovitch M. Molecular pathogenesis of pulmonary arterial hypertension. J Clin Invest 2012;122:4306-13.

11. Stenmark KR, Meyrick B, Galie N, Mooi WJ, McMurtry IF. Animal models of pulmonary arterial hypertension: the hope for etiological discovery and pharmacological cure. Am J Physiol Lung Cell Mol Physiol 2009;297:L1013-32.

12. Abe K, Toba M, Alzoubi A, Ito M, Fagan KA, Cool CD, et al. Formation of plexiform lesions in experimental severe pulmonary arterial hypertension. Circulation 2010; 121:2747-54.

13. Jasińska-Stroschein M, Owczarek J, Wesołowska A, Orszulak-Michalak D. Rosuvastatin, Sildenafil and their combination in monocrotaline-induced pulmonary hypertension in rat. Acta Pharm 2014;64:345-53.

14. Sheehan DC, Hrapchak BB. Theory and practice of histotechnology. Columbus: Battelle Press, 1980. 